\title{
CONVEXITY PROPERTIES OF THE MOMENT MAP FOR CERTAIN NON-COMPACT MANIFOLDS
}

\author{
ELISA PRATO
}

\begin{abstract}
Let $M$ be a connected symplectic manifold on which a torus $T$ acts in a Hamiltonian fashion and denote by $\Phi$ the corresponding moment map. If $M$ is compact, the Atiyah, GuilleminSternberg theorem says that the set $\Phi(M)$ is convex; if $M$ is noncompact this may not be the case. We show that if $M$ is suitably convex, then $\Phi(M)$ can be explicitly described as the convex hull of a finite number of affine rays. As an application we obtain a natural symplectic proof of the Paneitz convexity theorem for the positive Kaehler elliptic co-adjoint orbits.
\end{abstract}

\section{INTRODUCTION}

Let $M$ be a $2 m$-dimensional connected symplectic manifold with fundamental 2 -form $\omega$. Consider a torus $T$ with Lie algebra $\mathfrak{t}$. Assume that $T$ acts on $M$ in a Hamiltonian fashion and denote by $\Phi: M \rightarrow \mathfrak{t}^{*}$ the corresponding moment map; the pair $(M, T)$ is a Hamiltonian $T$-space. The convexity theorem, proven independently by Atiyah [A] and Guillemin-Sternberg [GS], says that, if $M$ is compact, the image set $\Phi(M)$ is the convex hull of the image via $\Phi$ of the $T$-fixed point set. If the manifold $M$ is not compact, $\Phi(M)$ may not be convex.

Counterexample 0.1 . Consider $\mathbb{C}^{2}$ with the canonical symplectic form. The two dimensional Cartan, $T$, of $U(2)$ acts on $\mathbb{C}^{2}$ by $\left(e^{i \theta_{1}}, e^{i \theta_{2}}\right) \cdot\left(z_{1}, z_{2}\right)=$ $\left(e^{-i \theta_{1}} z_{1}, e^{-i \theta_{2}} z_{2}\right)$; this action is Hamiltonian, the moment map being given

Current Address: DMI, Ecole Normale Supérieure, 45 Rue d'Ulm, 75230 Paris Cedex 05, France 


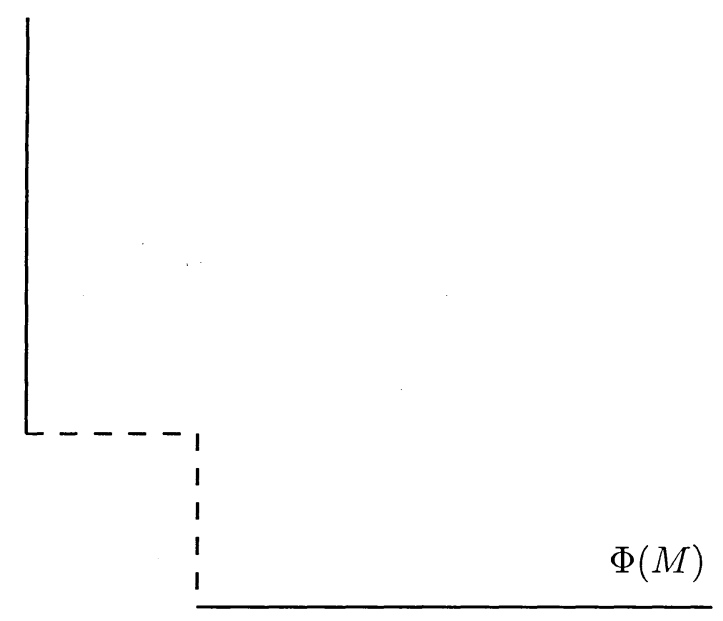

Figure 1. $\Phi\left(\mathbb{C}^{2}-\left(D^{1} \times D^{1}\right)\right)$ is not convex

by:

$$
\begin{aligned}
& \Phi: \mathbb{C}^{2} \longrightarrow \mathbb{R}^{2} \\
& \left(z_{1}, z_{2}\right) \longrightarrow \frac{1}{2}\left(\left|z_{1}\right|^{2},\left|z_{2}\right|^{2}\right) .
\end{aligned}
$$

Let now $M=\mathbb{C}^{2}-\left(D^{1} \times D^{1}\right), D^{1}$ denoting the closed unit disc in $\mathbb{C} . M$ is a connected symplectic manifold. The action of $T$ on $\mathbb{C}^{2}$ restricts to a Hamiltonian action on $M$. The corresponding moment map is the restriction of $\Phi$ to $M$, which we will still denote by $\Phi$. The set $\Phi(M)=\mathbb{R}_{+}^{2}-\{(x, y)$ s.t. $x \leq$ $\frac{1}{2}$ and $\left.y \leq \frac{1}{2}\right\}$ is not convex (Figure 1 ).

However, in many interesting situations (see examples below) the image set is still convex. From now on we will make an additional assumption. For each $X \in \mathfrak{t}$ denote by $\Phi_{X}$ the $X$-component of the moment map: $\Phi_{X}(p)=$ $\langle\Phi(p), X\rangle, p \in M$.

Assumption 0.2. There exists an integral element $X_{0} \in \mathfrak{t}$ such that $\Phi_{X_{0}}$ is a proper function having a minimum as its unique critical value.

REMARK 0.3. Since $X_{0}$ is integral, it generates a circle $S^{1}$ in $T$ whose induced action on $M$ is Hamiltonian with moment map $\Phi_{X_{0}}$. Notice that the 
requirement that $X_{0}$ is integral is not at all restrictive, since this can always be achieved by a small perturbation.

The goal of this article is to show that, under this assumption, the image set $\Phi(M)$ can be explicitly described as the convex hull of a finite number of affine rays in $\mathfrak{t}^{*}$ stemming from the images of $T$-fixed points (see Theorem 1.4 below.)

Example 0.4. Consider again $\mathbb{C}^{2}$ with the canonical symplectic form and the action of the Cartan, $T$, of $U(2)$. Take now the vector $X_{0}=(1,1) \in \mathfrak{t}$ generating the diagonal circle in $T$; the corresponding component of the moment map is

$$
\begin{aligned}
\Phi_{X_{0}}: \quad \mathbb{C}^{2} & \longrightarrow \mathbb{R} \\
\left(z_{1}, z_{2}\right) & \longrightarrow \frac{1}{2}\left(\left|z_{1}\right|^{2}+\left|z_{2}\right|^{2}\right) .
\end{aligned}
$$

It is immediately seen that $\Phi_{X_{0}}$ is a proper function whose unique critical value is the minimum 0 . Here $\Phi(M)=\mathbb{R}_{+}^{2}$.

EXAMPLE 0.5. Consider the upper sheet of the hyperboloid of two sheets:

$$
\mathcal{H}=\left\{(x, y, z) \in \mathbb{R}^{3} \mid z=\sqrt{1+x^{2}+y^{2}}\right\} .
$$

$\mathcal{H}$ is a connected symplectic manifold; in hyperbolic coordinates

$$
\left\{\begin{array}{l}
x=\sinh t \cos s \\
y=\sinh t \sin s \\
z=\cosh t
\end{array}\right.
$$

and the symplectic form is given by $\omega=\sinh t d s \wedge d t$. Here $T=S^{1}$ acts on $\mathcal{H}$ by rotation around the $z$-axis. It is easy to check that this action is Hamiltonian with moment map given by the projection onto the $z$-axis; this function is indeed proper and the minimum 1 is its unique critical value. Here $\Phi(\mathcal{H})=[1, \infty)$. This last assertion is just a fancy way of saying that the antisymmetric part, $\left(\begin{array}{cc}0 & z \\ -z & 0\end{array}\right)$, of all traceless $2 \times 2$ matrices with determinant 1 satisfies $|z| \geq 1$. In fact $\mathcal{H}$ is a positive Kaehler elliptic co-adjoint orbit of $S l(2, \mathbb{R})$ and $\Phi$ is the projection onto the dual of the compact Cartan subalgebra. We will discuss the projections of positive Kaehler elliptic orbits of more general non-compact Lie groups in Section 2. 
Acknowledgement. Our initial motivation came from [P], where Stephen Paneitz proves a convexity theorem for the positive Kaehler elliptic co-adjoint orbits. I am grateful to Victor Guillemin for his invaluable guidance. Finally, I wish to thank Regina Souza and Siye Wu for many helpful comments.

\section{Convexity}

1.1. Preliminaries. We recall that the moment mapping, $\Phi$, is a $T$-equivariant mapping satisfying the equations:

$$
d \Phi_{X}=\imath(\hat{X}) \omega \quad X \in \mathfrak{t},
$$

where $\widehat{X}$ is the vector field on $M$ with infinitesimal generator $X$. Let $p \in M$; denote by $T_{p}$ the stabilizer group of $p$ in $T$ and by $\mathfrak{t}_{p}$ the stabilizer algebra. One of the basic consequences of (1) is

Lemma 1.1. [GS] The image of $d \Phi_{p}$ is the annihilator space, $\mathfrak{t}_{p}^{\perp}$, of $\mathfrak{t}_{p}$ in $t^{*}$.

If we apply Lemma 1.1 to the induced action of $S^{1}$ we get that, if $p$ is regular for $\Phi_{X_{0}} X_{0}$ is not in $\mathfrak{t}_{p}$, and $\operatorname{dim} \mathfrak{t}_{p}^{\perp} \geq 1$. Consider now

$$
M^{1}=\left\{p \in M: \Phi_{X_{0}}(p) \text { regular, } \operatorname{dim} \mathfrak{t}_{p}^{\perp}=1\right\} .
$$

$M^{1}$ is a highly disconnected submanifold of $M$ ([GS], Theorem 3.5.). Consider, for each $p \in M^{1}$, the vector $v_{p}$ in $\mathfrak{t}_{p}^{\perp}$ satisfying

$$
\left\langle v_{p}, X_{0}\right\rangle=1 .
$$

It will be crucial for us to notice that for points in the same connected component of $M^{1}, \mathfrak{t}_{p}$, and therefore $v_{p}$, remain constant. Choose now a $T$-invariant metric on $M$, denote by $X_{0}$ the gradient of $\Phi_{X_{0}}$ and by $f_{t}$ its flow; it is not restrictive to assume that away from the critical value we have $d \Phi_{X_{0}}\left(X_{0}\right)=1$. Therefore, again by Lemma 1.1, we may assume

$$
d \Phi_{p}\left(X_{0}\right)=v_{p}, \quad p \in M^{1} .
$$

Since the metric is $T$-invariant, $f_{t}$ commutes with the action of $T$ and therefore preserves each connected component of $M^{1}$. 
Lemma 1.2.

$$
\Phi\left(f_{t}(p)\right)=\Phi(p)+t v_{p}, \quad p \in M^{1} .
$$

Proof. From (3)

$$
\frac{d}{d t} \Phi\left(f_{t}(p)\right)=[d \Phi]_{f_{\iota}(p)}\left(X_{0}\right)=v_{f_{t}(p)}=v_{p} .
$$

1.2. Statement and proof of the main result. Assume that $Y$ is a compact symplectic manifold and that a torus $H$ acts on it in a Hamiltonian fashion; denote by $\Psi$ the corresponding moment map. The image set $\Psi(Y)$ is beautifully characterized in the Atiyah, Guillemin-Sternberg convexity theorem, which we recall. Denote by $Y^{H}$ the set of points that are fixed by $H$.

Theorem 1.3. [A, GS] $Y^{H}$ is non-empty, $\Psi\left(Y^{H}\right)$ is finite, and $\Psi(Y)=$ $\mathcal{C} H \Psi\left(Y^{H}\right)$.

Here and in the following $\mathcal{C H}$ stands for convex hull. Consider now our original manifold $M$ and denote by $a$ the minimum value of $\Phi_{X_{0}}$; since $\Phi_{X_{0}}$ is proper, for each $s \geq a$ the $T$-invariant space $M_{s}=\Phi_{X_{0}}^{-1}(s)$ is compact. Assume for a moment that $S^{1}$ acts freely on $M_{s}, s>a$. Then the MardsenWeinstein reduced space, $Y_{s}=M_{s} / S^{1}$, is a compact symplectic manifold, and the induced action of $H=T / S^{1}$ is Hamiltonian. The corresponding moment map, $\Psi$, is characterized by $\Psi \circ \pi=q \circ \Phi$, where $\pi: M_{s} \rightarrow Y_{s}$ and $q: \mathfrak{t}^{*} \rightarrow \mathfrak{h}^{*}$ denote the projections. By Theorem 1.3 we then have that the $H$-fixed point set $Y_{s}^{H}$ is non-empty, $\Psi\left(Y_{s}^{H}\right)$ is finite, and

$$
q\left(\Phi\left(M_{s}\right)\right)=\Psi\left(Y_{s}\right)=\mathcal{C} H \Psi\left(Y_{s}^{H}\right) .
$$

But if we consider the set $M_{s}^{1}=M_{s} \cap M^{1}$ we have $\pi\left(M_{s}^{1}\right)=Y_{s}^{H}$ and in conclusion

$$
\Phi\left(M_{s}\right)=\mathcal{C} H \Phi\left(M_{s}^{1}\right) .
$$

Notice, incidentally, that we have also shown that the set $M^{1}$ is non-empty. If $S^{1}$ does not act freely on $M_{s}, Y_{s}$ is a $V$-manifold or orbifold. Such manifolds, though not necessarily smooth, do carry enough differentiable structures and a well defined symplectic form (see [W]); in addition, all of the properties that 
were needed above still hold. Fix now $u \in(a, \infty)$. Consider, for $p \in M_{u}^{1}$, the affine rays in $\mathfrak{t}^{*}$

$$
\alpha_{p}=\left\{\Phi(p)+t v_{p}: t \in[a-u, \infty)\right\}
$$

The choice of a regular $u$ here is completely arbitrary since $v_{p}$ does not depend on the choice of $p$ on the same gradient trajectory. Notice that there are only finitely many distinct $\alpha_{p}$ 's, each of which stems from the image of a fixed point for the action of $T$; in particular the $T$-fixed point set in $M$ is non-empty. We are now ready to prove:

\section{Theorem 1.4.}

$$
\Phi(M)=\mathcal{C} H\left\{\alpha_{p}: p \in M_{u}^{1}\right\}
$$

Proof. Begin by noticing that

$$
\Phi(M)=\bigcup_{t=a-u}^{\infty} \Phi\left(M_{u+t}\right)
$$

For a moment let us restrict our attention to $u+t>a$ (regular). By our previous remarks, $\Phi\left(M_{u+t}\right)=\mathcal{C} H \Phi\left(M_{u+t}^{1}\right)$. However, if we consider the gradient flow $f_{t}$, we have

$$
M_{u+t}=f_{t}\left(M_{u}\right) \quad \text { and } \quad M_{u+t}^{1}=f_{t}\left(M_{u}^{1}\right) .
$$

Therefore, by Lemma 1.2,

$$
\begin{aligned}
\Phi\left(M_{u+t}\right) & =\mathcal{C} H\left\{\Phi\left(f_{t}(p)\right): p \in M_{u}^{1}\right\} \\
& =\mathcal{C} H\left\{\Phi(p)+t v_{p}: p \in M_{u}^{1}\right\} .
\end{aligned}
$$

Continuity then forces

$$
\Phi\left(M_{a}\right)=\mathcal{C} H\left\{\Phi(p)+(a-u) v_{p}: p \in M_{u}^{1}\right\}
$$

and in conclusion:

$$
\Phi(M)=\bigcup_{t=a-u}^{\infty} \mathcal{C} H\left\{\Phi(p)+t v_{p}: p \in M_{u}^{1}\right\}=\mathcal{C} H\left\{\alpha_{p}: p \in M_{u}^{1}\right\} .
$$




\section{The KaEhler Elliptic CO-ADJOINT ORBITS}

Let $(G, K)$ be an irreducible Hermitian symmetric pair. $G$ is a non-compact, simple, connected Lie group with Lie algebra $\mathfrak{g}$ and $K$ a maximal compact subgroup with Lie algebra $\mathfrak{k} ; K$ is connected and its center, $S^{1}$, is a circle. Let $T$ be a Cartan subgroup of $K$ and let $W$ denote the Weyl group for the pair $\left(\mathfrak{k}^{\mathbb{C}}, \mathfrak{t}^{\mathbb{C}}\right)$. It is always possible to choose a set of positive roots, $\Delta^{+}$, for the pair $\left(\mathfrak{g}^{\mathbb{C}}, \mathfrak{t}^{\mathbb{C}}\right)$ such that the positive non-compact roots, $\Delta_{n}^{+}$, are larger than the compact ones, $\Delta_{c}^{+}$. With this choice, the elements of $\Delta_{n}^{+}$agree on vectors of the one-dimensional center of $\mathfrak{k}$; as a consequence we have that $\Delta_{n}^{+}$is $W$ invariant and that $\langle\alpha, \beta\rangle \geq 0$ for each $\alpha, \beta \in \Delta_{n}^{+}$. Consider now the convex, proper, $W$-symmetric cone in $\mathfrak{t}$ :

$$
\mathcal{C}=\left\{\nu \in \mathfrak{t}: \alpha(\sqrt{-1} \nu)>0 \text { for } \alpha \in \Delta_{n}^{+}\right\} .
$$

It will be convenient for us to denote $(-1)$ times the Killing form by $\langle$,$\rangle .$ We will use $\langle$,$\rangle to identify \mathfrak{g}$ with its dual, $\mathfrak{g}^{*}$; then $\mathfrak{k}$ and $\mathfrak{t}$ will be identified with $\mathfrak{k}^{*}$ and $\mathfrak{t}^{*}$, respectively, and adjoint orbits with the corresponding coadjoint orbits. An elliptic orbit is, by definition, an orbit that intersects $\mathfrak{t}$. Let $\lambda$ be an element of $\mathfrak{t}$ that lies in the cone $\mathcal{C}$ and let $\mathcal{O}_{\lambda}$ be the elliptic orbit $G \cdot \lambda$. The Kostant-Kirillov symplectic form gives $\mathcal{O}_{\lambda}$ the structure of a positive definite Kaehler manifold (see [Pr].)

The natural action of $T$ on $\mathcal{O}_{\lambda}$ is Hamiltonian with moment map, $\Phi: \mathcal{O}_{\lambda} \rightarrow$ $\mathfrak{t}$, given by the restriction to $\mathcal{O}_{\lambda}$ of the $T$-invariant projection of $\mathfrak{g}$ onto $\mathfrak{t}$. Let $X_{0}$ be the unique vector in the center of $\mathfrak{k}$ that satisfies $\alpha\left(\sqrt{-1} X_{0}\right)=1$ for $\alpha \in \Delta_{n}^{+}$(recall that the $\alpha^{\prime} s$ agree on the center); $X_{0}$ generates the center $S^{1}$. Our goal is to show that $S^{1}$ is the special circle of Assumption 0.2, and therefore that the projection $\Phi\left(\mathcal{O}_{\lambda}\right)$ is convex. An explicit description of this set, matching the one first given by Paneitz $[\mathrm{P}]$, is given in Theorem 2.4. First we need to recall certain basic facts from the structure theory of Hermitian symmetric spaces; we refer to $[\mathrm{H}, \mathrm{K} 1, \mathrm{~K} 2]$ for a more extensive treatment.

2.1. A review of Hermitian symmetric spaces. Let $\mathfrak{p}$ be the orthogonal complement of $\mathfrak{k}$ in $\mathfrak{g}$ with respect to $\langle,\rangle ; \mathfrak{g}=\mathfrak{k} \oplus \mathfrak{p}$ is the Cartan decomposition of $\mathfrak{g}$. The diffeomorphism of $K \times \mathfrak{p}$ onto $G$, given by $(k, X) \rightarrow e^{X} k$ induces a 
$K$-equivariant diffeomorphism

$$
\mathcal{O}_{\lambda} \simeq K \cdot \lambda \times \mathfrak{p}
$$

For each $\alpha \in \Delta_{n}^{+}$define $X_{\alpha} \in \mathfrak{t}$ by

$$
\left\langle X_{\alpha}, \nu\right\rangle=\frac{\alpha(\sqrt{-1} \nu)}{\langle\alpha, \alpha\rangle}, \quad \nu \in \mathfrak{t} .
$$

Notice that $\left\langle X_{\alpha}, X_{\beta}\right\rangle \geq 0, \alpha, \beta \in \Delta_{n}^{+}$, and that the convex hull of all rays $\left\{t X_{\alpha}: t \geq 0\right\}, \alpha \in \Delta_{n}^{+}$, is the closed proper cone, $\mathcal{C}^{*}$, dual to $\mathcal{C}$. Moreover, it is always possible to choose basis vectors $E_{\alpha}, F_{\alpha}$ for $\mathfrak{p}$ with the following properties

$$
\begin{gathered}
{\left[E_{\alpha}, X_{\alpha}\right]=2 F_{\alpha}, \quad\left[F_{\alpha}, X_{\alpha}\right]=-2 E_{\alpha}, \quad\left[E_{\alpha}, F_{\alpha}\right]=2 X_{\alpha}} \\
{\left[E_{\alpha}, X_{0}\right]=F_{\alpha}, \quad\left[F_{\alpha}, X_{0}\right]=-E_{\alpha} .}
\end{gathered}
$$

Note that from (6) follows easily that $S^{1}$ acts freely on $\mathfrak{p}-0$. Also, there exists a maximal subset, $\Psi$, of strongly orthogonal positive non-compact roots with the property that $\mathfrak{a}=\sum_{\alpha \in \Psi} \mathbb{R} E_{\alpha}$ is a maximal abelian subalgebra of $\mathfrak{p}$. If we consider the $\operatorname{subgroup} A=\exp (\mathfrak{a})$, then there is another very useful decomposition: $G=K A K$.

Denote now by $\pi$ the orthogonal projection $\mathfrak{g} \rightarrow \mathfrak{k}$. Then we have the following crucial lemma:

\section{Lemma 2.1.}

$$
\pi\left(a \cdot X_{0}\right)=X_{0}+\frac{1}{2} \sum_{\alpha \in \Psi}\left(\cosh 2 e_{\alpha}-1\right) X_{\alpha}
$$

where $a=e^{H}, H=\sum_{\alpha \in \Psi} e_{\alpha} E_{\alpha}$.

Proof.

$$
\begin{aligned}
\pi\left(a \cdot X_{0}\right) & =\pi\left(e^{a d H} \cdot X_{0}\right) \\
& =\pi\left(\sum_{k=0}^{\infty} \frac{a d^{k}(H) X_{0}}{k !}\right) \\
& =X_{0}+\sum_{k=1}^{\infty} \frac{a d^{2 k}(H) X_{0}}{(2 k) !}
\end{aligned}
$$




$$
=X_{0}+\frac{1}{2} \sum_{\alpha \in \Psi}\left(\cosh 2 e_{\alpha}-1\right) X_{\alpha},
$$

where the last equality follows from an easy computation using (5), (6).

2.2. Projecting the orbits. At this point we have all the information we need to show that $\mathcal{O}_{\lambda}$ satisfies Assumption 0.2 . We start by proving

Proposition 2.2. The component $\Phi_{X_{0}}$ is a proper function.

Proof. Since $X_{0}$ is in the center of $\mathfrak{k}$ we have, using the $K A K$ decomposition of $G$ :

$\Phi_{X_{0}}\left(\mathcal{O}_{\lambda}\right)=\Phi_{X_{0}}(K A K \cdot \lambda)=\left\langle K A K \cdot \lambda, X_{0}\right\rangle=\left\langle K \cdot \lambda, A \cdot X_{0}\right\rangle=\left\langle K \cdot \lambda, \pi\left(A \cdot X_{0}\right)\right\rangle$.

By Lemma $2.1 \pi\left(A \cdot X_{0}\right) \subseteq \mathcal{C}^{*}$. Since $\mathcal{C}$ lies entirely in $\mathfrak{t}$, one may replace $K \cdot \lambda$ with its projection onto $\mathfrak{t}, \Phi(K \cdot \lambda)$. It will now be enough to show that this last set is also contained in $\mathcal{C}$. By the Kostant convexity theorem, [Ko], $\Phi(K \cdot \lambda)$ is given by the convex hull of the set $W \cdot \lambda$; but $\mathcal{C}$ is convex and $W$-symmetric, and since $\lambda$ is in $\mathcal{C}$ so is all of $\Phi(K \cdot \lambda)$.

Proposition 2.3. $\Phi_{X_{0}}(\lambda)$ is the unique critical value of $\Phi_{X_{0}}$ and a minimum.

Proof. By Lemma 1.1 a point is critical for $\Phi_{X_{0}}$ if and only if it is fixed by the center of $K, S^{1}$. Therefore, in view of the $K$-equivariant diffeomorphism (4), and since $S^{1}$ acts freely on $\mathfrak{p}-0$, the critical set of $\Phi_{X_{0}}$ consists of the orbit $K \cdot \lambda$; this set maps to the unique value $\left\langle\lambda, X_{0}\right\rangle$. To show that this value is a minimum we argue as in Proposition 2.2 and we get, for $g=k a h$ ( $a \in A$, $h, k \in K)$

$$
\Phi_{X_{0}}(g \cdot \lambda)=\left\langle\Phi(h \cdot \lambda), \pi\left(a^{-1} \cdot X_{0}\right)\right\rangle .
$$

Finally, by Lemma 2.1 we have

$$
\Phi_{X_{0}}(g \cdot \lambda)=\left\langle\lambda, X_{0}\right\rangle+\frac{1}{2} \sum_{\alpha \in \Psi}\left(\cosh 2 e_{\alpha}-1\right)\left\langle\Phi(h \cdot \lambda), X_{\alpha}\right\rangle,
$$

where the second term on the left hand-side is non-negative since $\Phi(h \cdot \lambda) \in$ C.

We can finally apply Theorem 1.4 to get 


\section{Theorem 2.4.}

$$
\Phi\left(\mathcal{O}_{\lambda}\right)=\mathcal{C} H\left\{\bigcup_{w \in W}\left(w \cdot \lambda+\mathcal{C}^{*}\right)\right\}
$$

Proof. A direct computation using (5) shows that, for our orbits, the set of regular points with codimension-one stabilizer is

$$
M^{1}=\left\{e^{V_{\alpha}} w \cdot \lambda: \quad 0 \neq V_{\alpha} \in \operatorname{span}\left(E_{\alpha}, F_{\alpha}\right), \quad \alpha \in \Delta_{n}^{+}, \quad w \in W\right\} .
$$

In addition, for $p=e^{V_{\alpha}} w \cdot \lambda, \mathfrak{t}_{p}^{\perp}=\operatorname{span}\left(X_{\alpha}\right)$; therefore $v_{p}=X_{\alpha}$ and the rays are given by $\alpha_{p}=\left\{w \cdot \lambda+t X_{\alpha}: \quad t \geq 0\right\}, w \in W, \alpha \in \Delta_{n}^{+}$. Now apply Theorem 1.4, using the fact that the vectors $X_{\alpha}, \alpha \in \Delta_{n}^{+}$, generate $\mathcal{C}^{*}$.

We conclude with two pictures of the image set, each relative to a generic orbit of a rank-two group. The difference in the image set amounts to a different structure of the corresponding root system.

EXAmple 2.5. Consider

$$
G=S p(2, \mathbb{R})=\left\{g \in G l(4, \mathbb{R}): g^{t r}\left(\begin{array}{cc}
0 & I_{2} \\
-I_{2} & 0
\end{array}\right) g=\left(\begin{array}{cc}
0 & I_{2} \\
-I_{2} & 0
\end{array}\right)\right\}
$$

then

$$
K=\left\{\left(\begin{array}{cc}
A & B \\
-B & A
\end{array}\right) \in G l(4 ; \mathbb{R}): A+\sqrt{-1} B \in U(2)\right\} \simeq U(2),
$$

and

$$
\mathfrak{t}=\left\{\nu=\left(\begin{array}{cccc}
0 & 0 & x & 0 \\
0 & 0 & 0 & y \\
-x & 0 & 0 & 0 \\
0 & -y & 0 & 0
\end{array}\right):(x, y) \in \mathbb{R}^{2}\right\} \simeq \mathbb{R}^{2}
$$

With this last identification in mind, the one-dimensional center of $\mathfrak{k}$ is the $x=y$ line, and the compact Weyl group, $W$, acts by symmetry with respect to this axis. It is not difficult to check that the positive non-compact roots are $\alpha_{1}(\sqrt{-1} \nu)=2 x, \alpha_{2}(\sqrt{-1} \nu)=2 y$, and $\alpha_{3}(\sqrt{-1} \nu)=x+y$, and that $X_{\alpha_{1}}=(1,0), X_{\alpha_{2}}=(0,1)$, and $X_{\alpha_{3}}=(1 / 2,1 / 2)$. Therefore we have that $\mathcal{C}^{*}=\overline{\mathcal{C}}$ is the first quadrant. In Figure 2 we have drawn the image set for an orbit through a generic $\lambda \in \mathcal{C}$, according to Theorem 2.4. 


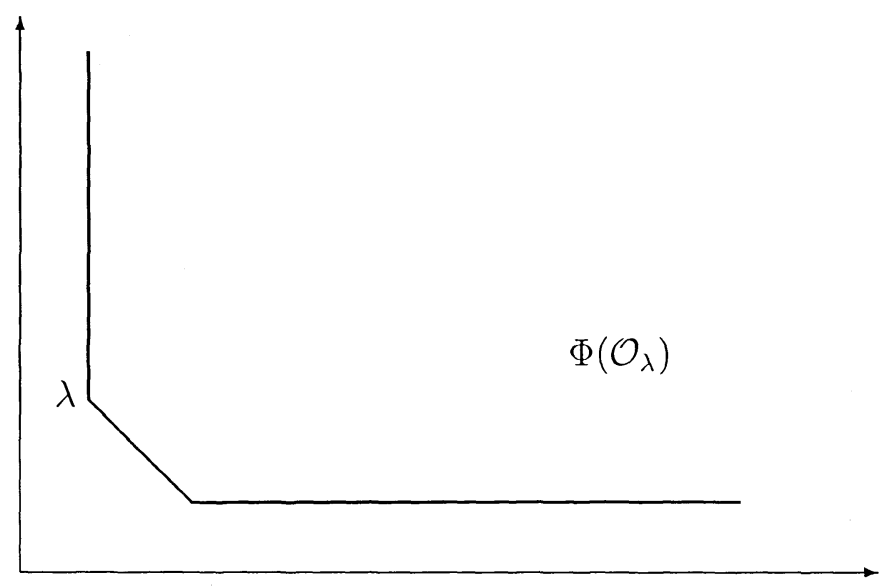

Figure 2. The $S p(2, \mathbb{R})$ case.

EXAMPLE 2.6. We get a slightly different picture in the case that

$$
\begin{gathered}
G=S U(2,1)=\left\{g \in S l(3 ; \mathbb{C}): \overline{g^{t r}}\left(\begin{array}{cc}
I_{2} & 0 \\
0 & -1
\end{array}\right) g=\left(\begin{array}{cc}
I_{2} & 0 \\
0 & -1
\end{array}\right)\right\}, \\
K=S(U(2) \times U(1))=\left\{\left(\begin{array}{cc}
A & 0 \\
0 & z
\end{array}\right): A \in U(2), z \in U(1), z \operatorname{det}(A)=1\right\},
\end{gathered}
$$

and

$$
\mathfrak{t}=\left\{\nu=\left(\begin{array}{ccc}
-\sqrt{-1} x & 0 & 0 \\
0 & -\sqrt{-1} y & 0 \\
0 & 0 & \sqrt{-1}(x+y)
\end{array}\right):(x, y) \in \mathbb{R}^{2}\right\} \simeq \mathbb{R}^{2} .
$$

As in the previous example, the one-dimensional center of $\mathfrak{k}$ is the $x=y$ line, and $W$ acts by symmetry about this axis. Here there are only two positive non-compact roots, $\alpha_{1}(\sqrt{-1} \nu)=x+2 y$ and $\alpha_{2}(\sqrt{-1} \nu)=y+2 x$; with our choice of coordinates, $X_{\alpha_{1}}=(1,0)$ and $X_{\alpha_{2}}=(0,1)$. Again $\mathcal{C}^{*}$ is the first quadrant. The difference here is that $\mathcal{C} \supset \mathcal{C}^{*}$; this gives us a greater freedom of choice for $\lambda$. In Figure 3 we have deliberately chosen $\lambda$ outside of $\mathcal{C}^{*}$.

\section{REFERENCES}

[A] Atiyah, M., Convexity and commuting Hamiltonians, Bull. London Math. Soc. 14 (1982), 1-15. 


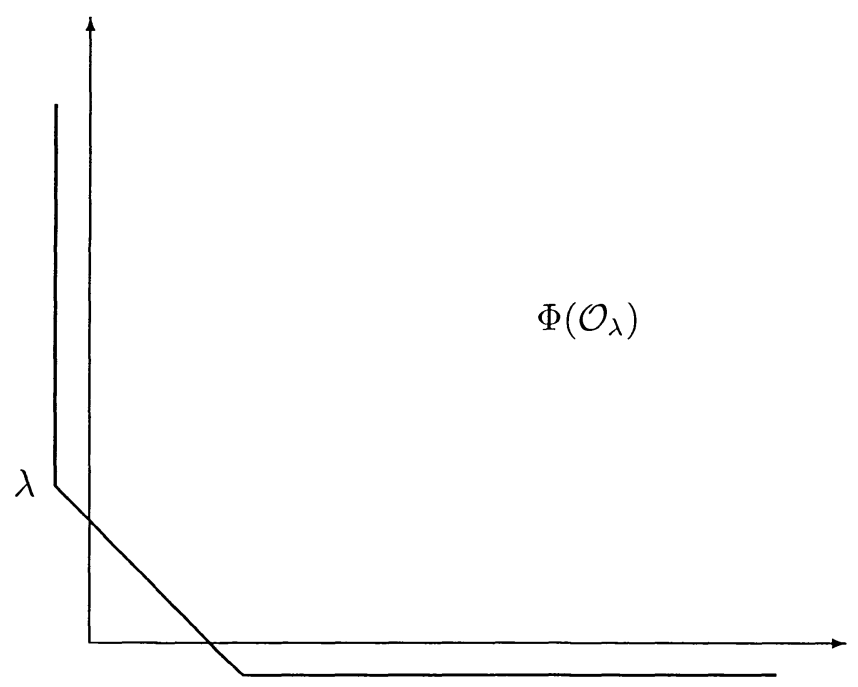

Figure 3. The $S U(2,1)$ case.

[GS] Guillemin, V. and Sternberg, S., Convexity properties of the moment mapping, Invent. Math. 67 (1982), 491-513.

[H] Helgason, S., Differential geometry, Lie groups and symmetric spaces, Academic Press, New York 1978.

[K1] Knapp, A., Bounded symmetric domains and holomorphic discrete series, in Symmetric Spaces, Marcel Dekker, Inc. 1972.

[K2] Knapp, A., Representation theory of semisimple Lie groups, Princeton Univ. Press 1986.

[Ko] Kostant, B., On convexity, the Weyl group, and the Iwasawa decomposition, Ann. Sci. Éc. Norm. Sup. 6 (1973), 413-455.

[P] Paneitz, S., Invariant convex cones and causality in semisimple Lie algebras and groups, J. Funct. Anal. 43 (1981), 313-359.

[Pr] Prato, E., Some symplectic invariants of Hamiltonian K-actions, Ph. D. thesis, M.I.T. 1990.

[W] Weinstein, A., Symplectic $V$-manifolds, periodic orbits of Hamiltonian systems, and the volume of certain Riemannian manifolds, Comm. Pure Appl. Math. 30 (1977), 265-271.

Princeton University, U. S. A.

Received DeCember 3, 1993 